\title{
Neoplasia Pseudopapilifera Sólida de Pâncreas (Tumor de Frantz): Relato de Caso
}

doi: https://doi.org/10.32635/2176-9745.RBC.2020v66n4.826

\author{
Solid Pseudopapillary Tumor of the Pancreas (Frantz's Tumor): Case Report \\ Neoplasia Sólida-Pseudopapilar de Páncreas (Tumor de Frantz): Relato de Caso
}

\author{
Carlos Rafael Lima Monção'; Levindo Tadeu Freitas de Figueiredo Dias²; Cecília Barbosa Alcântara3; Thereza Cristina Abreu Versiani; \\ Autieri Alves Correia ${ }^{5}$
}

\section{RESUMO}

Introduçáo: A neoplasia pseudopapilífera sólida de pâncreas (tumor de Frantz) é muito rara e representa menos de 1\% de todos os tumores pancreáticos. Relato do caso: Paciente do sexo feminino, 48 anos, internada para investigaçáo de massa intra-abdominal de etiologia a esclarecer. Foi submetida a tratamento cirúrgico com sucesso na ressecção tumoral e não apresentou complicaçôes no pós-operatório. Os pacientes usualmente tornam-se sintomáticos somente após longos períodos de evolução, quando então apresentam desconforto ou dor abdominal, ou a palpação de massas abdominais no exame físico. Para o diagnóstico, são necessários exames de imagem como ultrassonografia e tomografia computadorizada, que geralmente evidenciam volumosas massas heterogêneas intra-abdominais. É necessária a confirmação com estudo anatomopatológico e comumente estas possuem características histológicas compatíveis com tumores de baixo grau de malignidade. A ressecçáo cirúrgica é o tratamento recomendado e mesmo os tumores de grandes dimensóes possuem chances de curas consideráveis, se for possível obter margens cirúrgicas livres. Sabe-se que a ressecção tumoral completa é associada a longos períodos de sobrevida. Conclusáo: A neoplasia pseudopapilífera sólida de pâncreas (tumor de Frantz), apesar de rara, deve ser considerada no diagnóstico diferencial de tumores de pâncreas, já que o tratamento cirúrgico apresenta boas chances de cura mesmo em tumores de grandes dimensóes.

Palavras-chave: Neoplasias Pancreáticas; Neoplasias Abdominais; Pâncreas; Mulheres; Relatos de Casos.

\section{ABSTRACT}

Introduction: The solid pseudopapillary tumor of the pancreas (Frantz's tumor) is a neoplasm extremely rare that represents less than $1 \%$ of all the pancreatic tumors. Case report: 48 years old female patient was hospitalized with intra-abdominal mass of etiology hard to define. She was submitted to surgical treatment with successful laparotomy tumor resection and there were no postoperative complications. The patients usually become symptomatic only after long time of evolution, when they have discomfort or abdominal pain or palpation of abdominal masses during physical examination. Complementary exams like ultrasound and computed tomography are necessary to diagnosis and usually they show heterogeneous large intra-abdominal masses. It is necessary the confirmation with anatomopathological study which usually has histological characteristics compatible with low-grade malignancy. The surgical resection is the recommended treatment and even large size tumors has a considerable chance of cure if free surgical margins are obtained. It is known that complete tumoral resection is associated with long-term survival. Conclusion: The solid pseudopapillary tumor of the pancreas (Frantz's tumor), although rare, should be considered in differential diagnosis of pancreatic neoplasms because surgical treatment usually has good chances of cure even in large size tumors.

Key words: Pancreatic Neoplasms; Abdominal Neoplasms; Pancreas; Women; Case Reports.

\section{RESUMEN}

Introducción: La neoplasia sólida-pseudopapilar de páncreas (tumor de Frantz) es muy extraordinaria y representa menos de $1 \%$ de todos los tumores pancreáticos. Relato del caso: Paciente do sexo femenino, 48 años, internada para investigación de masa intraabdominal de etiología a esclarecer. Fue sometida al tratamiento quirúrgico con éxito en la resección tumoral y no presentó complicaciones en el postoperatorio. Los pacientes por lo general se convierten en sintomáticos solamente después de largos períodos de evolución, cuando presentan incomodidad o dolor abdominal o existe palpación de masas abdominales en el examen físico. Para el diagnóstico son necesarios exámenes de imagen como ecografía y tomografía computarizada que generalmente demuestran voluminosas masas heterogéneas intraabdominales. Es necesaria la confirmación con examen anatomopatológico y generalmente tienen características histológicas compatibles con tumores de poco grado de malignidad. La resección quirúrgica es el tratamiento indicado y mismo tumores de grandes dimensiones poseen posibilidades de curas considerables se sea posible obtener márgenes quirúrgicas libres. Se sabe que a resección tumoral completa es asociada a largos períodos de sobrevida. Conclusión: La neoplasia sólida-pseudopapilar de páncreas o tumor de Frantz: a pesar de extraordinaria, debe ser considerada en el diagnóstico diferencial de tumores de páncreas y a que el tratamiento quirúrgico presenta buenas posibilidades de cura, mismo en tumores de grandes proporciones.

Palabras clave: Neoplasias Pancreáticas; Neoplasias Abdominales; Páncreas; Mujeres; Informes de Casos.

${ }^{1}$ Hospital Universitário Clemente de Faria (HUCF). Montes Claros (MG), Brasil. Orcid iD: https://orcid.org/0000-0001-9784-9569 ${ }^{2}$ HUCF. Fundação Hospitalar Dilson Godinho. Montes Claros (MG), Brasil. Orcid iD: https://orcid.org/0000-0002-7524-8567 ${ }^{3}$ HUCF. Montes Claros (MG), Brasil. Orcid iD: https://orcid.org/0000-0003-0661-003X ${ }^{4}$ HUCF. Montes Claros (MG), Brasil. Orcid iD: https://orcid.org/0000-0002-6649-6550 ${ }^{5}$ HUCF. Montes Claros (MG), Brasil. Orcid iD: https://orcid.org/0000-0002-5948-0526 Endereço para correspondência: Carlos Rafael Lima Monção. Rua Tupinambás, 13, Sala 1312 - Melo. Montes Claros (MG), Brasil. CEP $39401-509$. E-mail: crmoncao.crm@gmail.com 


\section{INTRODUÇÃO}

Primeiramente descrita por Frantz em 1959, a neoplasia pseudopapilifera sólida de pâncreas é rara (representa $0,3 \%$ a $2,7 \%$ de todas as neoplasias pancreáticas); mais comum em mulheres jovens, em geral na terceira década de vida; e possui um baixo potencial de malignidade ${ }^{1,2}$. A presença de massas abdominais é a apresentação clínica mais comum. O tratamento é essencialmente cirúrgico e com boas chances de cura, já que se trata de uma neoplasia de lento crescimento tumoral ${ }^{3}$.

A metodologia utilizada foi baseada no modelo para elaboraçáo de relato de caso do Case report guidelines (CARE) com a descrição dos achados clínicos, a avaliação diagnóstica, a intervenção terapêutica e o seguimento clínico de casos raros ou de apresentação atípica ${ }^{4}$.

O presente estudo tem como objetivo realizar um relato de caso com descrição da história clínica, das alteraçôes no exame físico, das alteraçōes nos exames de imagem, além do tratamento cirúrgico, do estudo anatomopatológico e do acompanhamento no pós-operatório tardio de um tumor de incidência rara e, por isso, é de suma importância para alertar os profissionais de saúde sobre o diagnóstico do tumor de Frantz e a experiência clínica adquirida nesse caso.

\section{RELATO DO CASO}

Paciente do sexo feminino, 48 anos de idade, admitida no Hospital Universitário Clemente de Faria de Montes Claros, com queixa de dor abdominal com cinco meses de evolução. Caracterizava a dor como de moderada intensidade, localizada em hipocôndrio esquerdo e sem irradiação. O quadro era acompanhado de náuseas, hiporexia e perda ponderal de $5 \mathrm{~kg}$ em dois meses. O exame físico do abdome revelou massa em hipocôndrio esquerdo, dolorosa à palpação, móvel, endurecida, irregular e com bordas aparentemente lisas. A ultrassonografia de abdome evidenciava massa expansiva heterogênea com áreas hipoecogênicas de permeio em íntimo contato com a cauda do pâncreas, que a tomografia computadorizada mostrou tratar-se de volumosa massa de partes moles aparentemente capsulada, bem delimitada e contendo calcificaçóes parietais, apresentando realce irregular pelo meio de contraste, com áreas hipoatenuantes de permeio, localizada no hipocôndrio/flanco esquerdo, em íntimo contato com o corpo/cauda pancreática e câmara gástrica, medindo cerca de $12,1 \times 11,3 \times 10,0 \mathrm{~cm}$, compatível com neoplasia (Figura 1 e Figura 2), tendo como principais hipóteses diagnósticas o tumor de Frantz e a neoplasia estromal gastrintestinal (GIST).

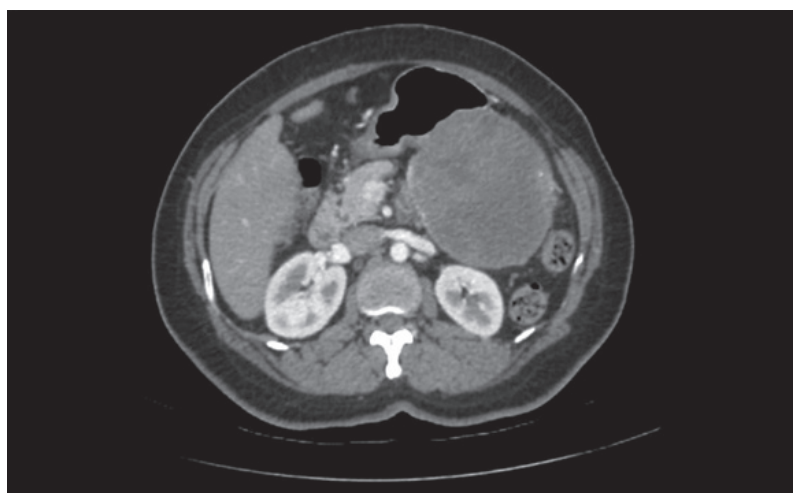

Figura 1. Tomografia computadorizada com imagem transversal do tumor

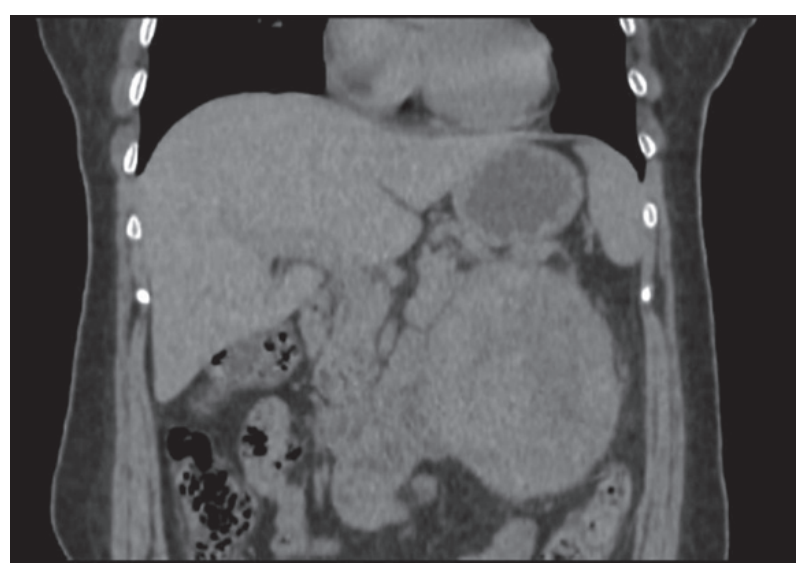

Figura 2. Tomografia computadorizada com imagem coronal do tumor

Realizada laparotomia com incisão subcostal bilateral. Ao inventário da cavidade, foi encontrada grande lesão tumoral acometendo corpo e cauda do pâncreas, com íntimo contato com a artéria e veia esplênica, não foi evidenciando implante peritoneal ou sinais de doença avançada. Foram feitas ressecção da lesão e ligadura da veia esplênica. O coto do pâncreas foi fechado com dupla linha de sutura (Figura 3).

Os achados anatomopatológicos revelaram, à macroscopia, formação tumoral medindo 12,0 x 11,0 x 8,5 cm e pesando 619 gramas; superfície externa pardacenta e bocelada, envolvida por pseudocápsula fibrosa com pequena área suspeita de extravasamento capsular; e, aos cortes, lesão amarronzada, friável, heterogênea com áreas císticas e hemorrágicas.

Ao exame histopatológico, confirmou-se neoplasia pseudopapilífera sólida do pâncreas (tumor de Frantz) com margens cirúrgicas livres. A paciente evoluiu sem intercorrências no pós-operatório. Está em acompanhamento com a oncologia clínica do Hospital Dílson Godinho, desde entâo, com consultas semestrais. Um ano após a cirurgia, apresentava-se assintomática, com exame físico e laboratoriais: hemograma, íons, função 


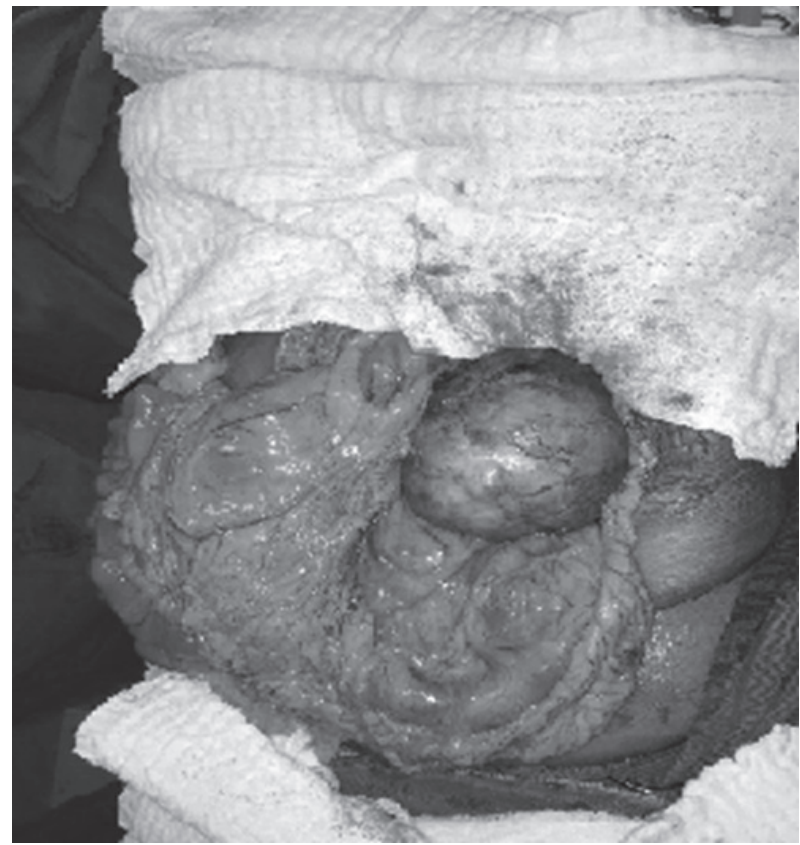

Figura 3. Laparotomia evidenciando lesão neoplásica

renal, função hepática, LDH, ferritina, CA19-9, CA125 e CEA, sem alteraçóes.

O presente relato de caso obteve aprovação do Comitê de Ética em Pesquisa das Faculdades Integradas Pitágoras de Montes Claros/Brasil, CAAE: 28326620.8.0000.5109.

\section{DISCUSSÃO}

Em 1959, Frantz descreveu três casos de tumores pancreáticos com características distintas das neoplasias de pâncreas mais comuns, principalmente por haver áreas císticas e sólidas dentro de um tumor encapsulado.

A partir de então, aproximadamente 800 casos foram descritos na literatura científica. Estima se que esses tumores sejam responsáveis por $0,3 \%$ a $2,7 \%$ de todas as neoplasias pancreáticas ${ }^{1,5}$. Usualmente acometem pacientes jovens, do sexo feminino, na terceira década de vida, em uma proporçáo de 10:1 $1^{6,7}$.

Os pacientes permanecem assintomáticos por longos períodos até que a apresentação clínica mais comum seja a presença de massa abdominal, muitas vezes de grandes dimensóes, acompanhada de dor abdominal e raramente icterícia ${ }^{2}$.

O tratamento é essencialmente cirúrgico. Essa neoplasia apresenta um bom prognóstico em razão do seu lento crescimento tumoral ${ }^{8}$. Mesmo os tumores de grandes dimensôes são passíveis de ressecção cirúrgica com chances de cura 9 . O tratamento cirúrgico pode envolver a necessidade de avaliação das ressecções vasculares para se obter margens cirúrgicas livres. Geralmente, não há indicação de linfadenectomia pela aparente ausência de invasão linfonodal desse tipo específico de neoplasia. Pode-se optar por cirurgia laparoscópica, dependendo da localização do tumor no parênquima pancreático, das dimensóes da neoplasia e da experiência da equipe. A complicação mais comum no pós-operatório são as fístulas pancreáticas 5 .

Outras modalidades terapêuticas adjuvantes, como quimioterapia e radioterapia, também já foram relatadas, porém sem obter conclusôes de forte evidência científica, uma vez que se trata de um tumor com poucos casos descritos e com boa resposta ao tratamento cirúrgico isolado ${ }^{10}$.

Após a ressecção cirúrgica completa, cerca de $95 \%$ dos pacientes apresentam sobrevida livre da doença após cinco anos. As recidivas tumorais são associadas principalmente a ressecçóes cirúrgicas com margens cirúrgicas positivas e usualmente são diagnosticadas por evidências de metástases hepáticas ou por recidiva no próprio parênquima pancreático ${ }^{11}$.

\section{CONCLUSÃO}

O relato de caso de neoplasia pseudopapilífera sólida do pâncreas, considerada rara, com poucas descrições na literatura científica, em uma paciente que foi submetida ao tratamento cirúrgico com sucesso, é importante para que outros profissionais médicos tenham conhecimento sobre esse tipo de tumor, com sua apresentação clínica, suas alteraçôes características nos exames de imagem e a descrição do tratamento cirúrgico realizado, compartilhando e descrevendo a experiência adquirida na condução desse caso.

\section{CONTRIBUIÇÕES}

Carlos Rafael Lima Monção contribuiu na concepção e/ou no planejamento do estudo; na obtenção, análise e interpretação dos dados; assim como na redação e revisão crítica. Levindo Tadeu Freitas de Figueiredo Dias e Cecília Barbosa Alcântara contribuíram na redação do manuscrito. Thereza Cristina Abreu Versiani e Autieri Alves Correia contribuíram na concepção e/ou no planejamento do estudo; e na obtenção, análise e interpretação dos dados. Todos os autores aprovaram a versão final a ser publicada.

\section{DECLARAÇÃO DE CONFLITO DE INTERESSES}

Nada a declarar.

\section{FONTES DE FINANCIAMENTO}

Não há. 


\section{REFERÊNCIAS}

1. Frantz VK. Tumors of the pancreas. Washington, DC: Armed Force Institute of Pathology; 1959. (Atlas of tumor pathology Section 7, fasc. 27-28).

2. Campos M, Campbell J, Gonzales H, et al. Tackling the diagnosis: solid pseudopapillary tumor of the pancreas in a young man. Gastroenterology Res. 2019;12(3):174-5. doi: https://doi.org/ 10.14740/gr1170

3. Llatas J, Palomino A, Frisancho O. Tumor de Frantz: neoplasia sólida pseudopapilar de páncreas. Rev Gastroenterol Perú. 2011;31(1):56-60.

4. Gagnier JJ, Kienle G, Altman DG, et al. The CARE guidelines: consensus-based clinical case reporting guideline development. J Med Case Reports.2013;7:223. doi: https://doi.org/10.1186/1752-1947-7-223

5. Carlotto JRM, Torrez FRA, Gonzales AM, et al. Solid pseudopapillary neoplasm of the pancreas. ABCD Arq Bras Cir Dig. 2016;29(2):93-6. doi: https://doi. org/10.1590/0102-6720201600020007

6. Affirul CA, Qisti FN, Zamri Z, et al. Pancreatic pseudopapillary tumour: a rare misdiagnosed entity. Int J Surg Case Rep. 2014;5(11):836-9. doi: https://doi. org/10.1016/j.ijscr.2014.09.021

7. Kallichanda N, Tsai S, Stabile BE, et al. Histogenesis of solid pseudopapillary tumor of the pancreas: the case for the centroacinar cell of origin. Exp Mol Pathol. 2006;81(2):101-7. doi: https://doi.org/10.1016/j. yexmp.2006.05.005

8. Del Chiaro M, Verbeke C, Salvia R, et al. European experts consensus statement on cystic tumours of the pancreas. Dig Liver Dis. 2013;45(9):703-11. doi: https:// doi.org/10.1016/j.dld.2013.01.010

9. Ud Din N, Arshad H, Ahmad Z. Solid pseudopapilllary neoplasm of the pancreas. A clinicopathologic study of 25 cases from Pakistan and review of Literature. Ann Diagn Pathol. 2014;18(6):358-62. doi: https://doi. org/10.1016/j.anndiagpath.2014.10.001

10. Fried P, Cooper J, Balthazar E, et al. A role for radiotherapy in the treatment of solid and papillary neoplasms of the pancreas. Cancer. 1985;56(12):2783-5. doi: https://doi. org/10.1002/1097-0142(19851215)56:12<2783::aidcncr2820561211>3.0.co;2-q

11. Patnayak R. et al. Solid and cystic papillary neoplasm of pancreas: a clinic-pathological and immunohistochemical study: a tertiary care center experience. South Asian J Cancer. 2013;2(3):153-7. doi: https://doi. org/10.4103/2278-330X.114141 